\title{
Escorting Students into Responsibility and Autonomy (ESRA): A Model for Supervising Degree Projects
}

\author{
Malin Jordal (iD) \\ Henrik Eriksson (iD) 2,3 \\ Martin Salzmann-Erikson (D) \\ Monir Mazaheri (iD) 2,4 \\ 'Department of Caring Sciences, \\ University of Gävle, Gävle, Sweden; \\ ${ }^{2}$ Department of Health Sciences, The \\ Swedish Red Cross University College, \\ Huddinge, Sweden; ${ }^{3}$ Department of \\ Health Sciences, University West, \\ Trollhättan, Sweden; ${ }^{4}$ Department of \\ Neurobiology, Care Sciences and Society, \\ Karolinska Institutet, Huddinge, Sweden
}

Correspondence: Malin Jordal Department of Caring Sciences, University of Gävle, Gävle, Sweden

Tel +46738593373

Email malin.jordal@hig.se
Background: Several models for how to support students and provide them with the skills needed to write their degree projects have been proposed. However, few attempts have been made to present a general model for students' academic work based on reasoning and communication skills rather than memorizing and mimicking their supervisors during their independent degree project.

Objective: In the present paper, we propose a well-structured model that assists supervisors in promoting students' responsibility and autonomy, while at the same time maintaining a high level of support.

Presentation: We present a step-by-step protocol based on a partnership model with a contractual style that focuses on students' academic work with their own texts through a process of alternating between abstract and concrete writing. This protocol, which is called the ESRA (Escorting the Students into Responsibility and Autonomy) model, can be utilized regardless of the content, specific aim and scope of the individual student's degree project.

Discussion and Conclusions: We argue that this model promotes high levels of engagement and assumption of responsibility among students, while also offering a feasible structure for ensuring the steps to empowerment and autonomy. Use of the ESRA model is suitable when a constructive interaction between students and supervisors is desirable as a tool to achieve the learning outcomes of the degree project. Thus, the proposed model is one step toward giving a new generation of nurses the skills and ability they need to adapt in the changing world of the 21 st century and to make promoting health a core mission of their profession.

Keywords: learning, nursing education, peer learning, supervision, writing

\section{Introduction}

The independent degree project is carried out by students under the supervision of tutors, whose roles are to facilitate students' learning process, encourage reflexive thinking and provide a platform for integrating the knowledge acquired into future academic or professional life. ${ }^{1}$ Writing a degree project also provides students with theoretical insights and functional knowledge, ${ }^{2}$ thus contributing to lifelong learning. ${ }^{3}$

Thus, the aim of the degree project is to equip students with the criticalthinking and problem-solving skills that not only prepare them for further academic studies, but for future professional work and life in general. Yet how to best support students' academic writing in their independent degree projects in nursing programs has been a debated didactic challenge for decades. $^{4-6}$ The philosophical and theoretical content of the nursing discipline 
is well-incorporated into academic nursing education. ${ }^{7}$ However, the academic setting that is to support nursing students in conducting their independent degree project and writing scientific texts is highly variable and sometimes inadequate. Previous studies have shown that nursing students feel their first years of education do not prepare them for developing scientific academic texts or for reflective writing with a sufficient level of academic rigor, ${ }^{8}$ but mainly for a synthetic type of writing required in clinical scenarios. ${ }^{9}$

Several models have been proposed for supporting students and providing them with the skills needed to write their degree projects. Dysthe ${ }^{10}$ divided the existing supervising and mentoring scenarios into three different models: the teaching model, the partnership model and the apprenticeship model. The teaching model defines a "traditional" teacher-student relationship with an emphasis on asymmetry, status difference, and dependency. The partnership model is more symmetrical: The student's thesis work is seen as a joint project between student and supervisor, and cooperation is emphasized that includes feedback through dialogue, where explanatory texts form the basis for discussion. The apprenticeship model is characterized by the student's learning by observing and performing tasks in the company of a supervisor or a "master" who assumes clear authority. Further, Gatfield ${ }^{1}$ described and labelled four different supervisory management styles: laissez-faire, pastoral, directorial and contractual. The laissez-faire style is defined by low structure and low support, where the supervisor may appear uncaring and noninterfering. The pastoral style is defined by low structure and high support, where the supervisor provides considerable personal care and support, but not necessarily any task-driven directives. The directorial style is defined by high structure and low support, where the supervisor has a close and regular interactive relationship with the candidate, but avoids non-task issues. The contractual style is defined by high structure and high support, where the supervisor offers direction, something that requires a highlevel management skills and good interpersonal relationships.

However, it may be that none of these models are entirely suited to the educational needs of the new generation of students. Frenk et $\mathrm{al}^{12}$ proposed that:

The education of health professionals in the 21th century must focus less on memorising and transmitting facts and more on promotion of the reasoning and communication skills that will enable the professional to be an effective partner, facilitator, adviser, and advocate. (p1945)

Today's students, growing up in an information culture with almost unlimited internet access, are comfortable with actively seeking information and less prone to accept traditional educational styles characterized by teacher superiority. Previous research on academic writing among nursing students has demonstrated that the implemented reforms in nursing education are too often considered an end goal rather than a starting point for what Frenk et $\mathrm{al}^{12}$ claimed to be important for the 21 th century $\left(\mathrm{cf}^{2,5}\right)$. Academic reforms have not changed the structure or the content of nursing education to any great extent. On the whole, nursing education is still aimed at getting students ready for their first day at work as nurses, missing out on the important aspect addressed by Frenk et al. ${ }^{12}$ The shortcomings as regards promoting reasoning and communication skills in education constitute a challenge that still needs to be resolved. We argue that what students need to achieve these goals is support in developing the specific writing skills required for academic writing, as well as skills in reflective, critical thinking, ${ }^{13}$ which would also improve their competence as professional nurses. There is thus a need for a model that appreciates and promotes high engagement among students and that embraces their responsibility for their own learning, without failing to provide them with structure and support. In the present paper, we propose a well-structured model that can assist supervisors in flatting their power horizon by promoting students' empowerment and independence, while at the same time maintaining a high level of support: the ESRAmodel. ESRA is an acronym for Escorting Students into Responsibility and Autonomy. It was developed to be suitable for group supervision of nursing students who are writing their degree project. The model emphasizes guiding or escorting students toward embracing authentic responsibility and in the end having the ability to defend their thesis with autonomy. The structure and buildup of ESRA also enables scientific interaction between students and supervisors as a means of knowledge development. Thus, the ESRA model provides a high level of support, through its strong emphasis on "escorting" and by providing a clear and well-designed structure for the process of writing a degree project. This structure, in turn, helps students navigate through and achieve the expected outcomes of the degree project. 


\section{Theoretical Framework: ESRA a Partnership Model with a Contractual Style}

To escort students into responsibility and autonomy, we preserve the role of supervisor as a facilitator; thus, the master-apprentice ${ }^{10}$ element of supervision is toned down, instead emphasizing partnership. Further, the model includes regular pedagogical elements, implemented at each tutorial/supervision meeting, that encourage students' reflective thinking as well as their autonomy in and responsibility for their own process. In the ESRA model, we start from the partnership model ${ }^{10}$ and the contractual style as defined by Gatfield, ${ }^{1}$ thus the model involves cooperation, dialogue using explanatory texts, and a high level of structure and support. Such an understanding or paradigm of supervision relates to a pedagogical philosophy aimed at consciously fostering students' independent and critical thinking and is built on dialogism, an epistemology that sees knowledge as emerging from interaction and the interpenetration of different voices (cf. ${ }^{14,15}$ ). It also relates to a constructivist pedagogy where knowledge is understood as something which is constructed, not transmitted, and where the emphasis is placed on students' active role as learners. Within such a pedagogical philosophy $^{16}$ students and supervisors become cocreators in achieving the desired learning objectives by engaging in constant dialogue and demonstrating their willingness to listen to each other and postpone judgment.

To work successfully based on this paradigm, a structure is needed that gives relevant directions toward the end goal. In the ESRA model, we propose a structure that focuses on students' academic work with texts. Unlike the writing one does as a clinical nurse, writing that is concrete and uses specific wording in relation to a person's care plan, academic writing has an overall structure alternating between abstract and concrete writing in the degree project regardless of content. Further, academic writing derives from certain rules that can be easily adapted to and, over time, remembered as a format. These didactic elements, the alternation between the abstract and concrete, symbolized using a symmetric $\mathrm{V}$ shape, and the application of certain academic rules are illustrated in Figure 1.

To educate and successfully supervise academic writing using the ESRA model, it is important to start from some key points that support crucial academic literacy skills, such as critical thinking, abstraction, reasoning and summing up. The symmetric V shape (Figure 1) symbolizes a structure of "touching down" or moving from the abstract to the concrete and then back to the abstract. This metaphor or symbolism is relevant to the scholarly research paper, where the top left of the $\mathrm{V}$ symbolizes the starting point and moving from "higher" abstraction in the introduction and background "downwards" to concrete writing in methods and results and, then, "up" again into abstraction in the discussion and conclusion. Thus, the ESRA model supports students' understanding of the continuous alternation between abstract and concrete in academic writing, as well as their skills in repeating a certain structure over and over again. The symmetric V-shaped formation furthermore illustrates the order of the didactic

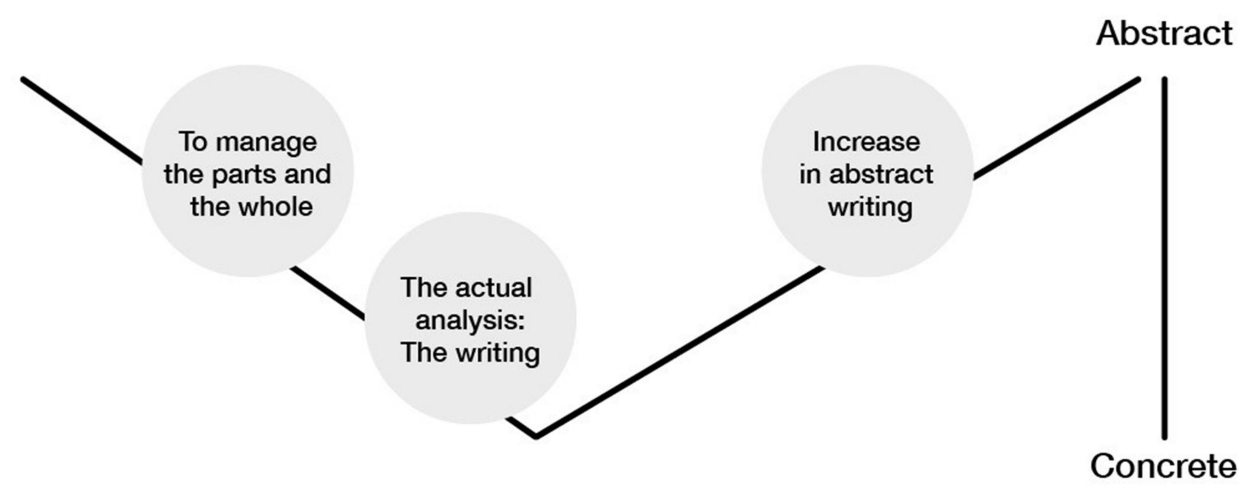

$0 \%$

Figure I The ESRA model: a symmetric V-shaped academic writing formation. 
activities, including where they are placed during the course of writing as well as in the scholarly research paper, something that further supports students' understanding of the alternation between abstract and concrete writing.

The ideal set-up for supervising a degree project using the ESRA model is to have two supervisors and 8-10 students working in pairs. The layout of the model involves a group of students meeting on four occasions. These four meetings are labelled the " $0 \%$, $40 \%, 70 \%$ and $90 \%$ meetings" demonstrating the assumed percentage of completion of the degree project at each meeting; each day is assigned a certain exercise (see Figure 2). The staircase also illustrates an "upward" movement toward a complete degree project. For the four days assigned for the meetings, the students will work full days $(9 \mathrm{am}-5 \mathrm{pm})$, whereas the supervisors, after introducing the day's exercise and getting the students started, will be present only in the afternoons. The time assigned for students without the presence of the supervisors allows the students to collaborate and supervise each other, which encourages responsibility and autonomy. The supervisors role in setting up the direction of the work, as well as assigning an exercise for each day and then reconnecting in the afternoon, demonstrates how they escort the students into responsibility and autonomy. Below, we describe the process and outline planning and activities in a typical supervision sequence following the ESRA model.

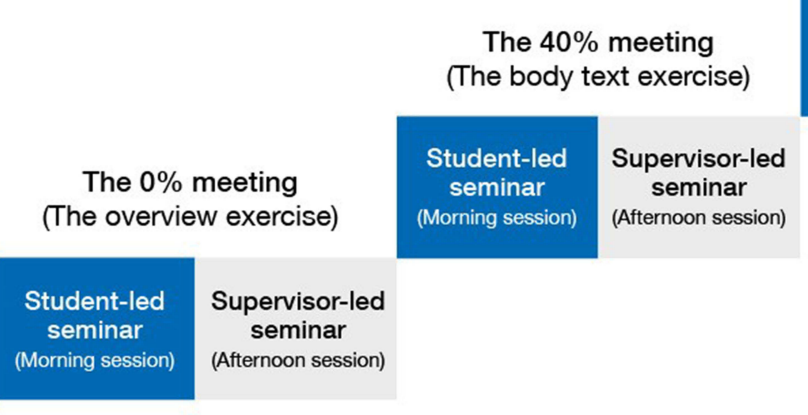

\section{The ESRA Model Supervision: Step- by-Step Prerequisites and Preparing for the Supervision}

The starting point of the proposed ESRA model is that eligible students must have passed a 15 ECTS course that includes examination of a degree project plan.

It is recommended that the supervisors provide a supervision schedule, including the meeting dates, two weeks prior to the course start. The students are expected to prepare themselves for every meeting by reading another student pair's texts-excerpts. It is advisable to let the students participate in distributing these texts as a way of introducing them to active participation, as well as to ensure that these texts rotate, so that all pairs are "exposed" to others' projects. The intervals between the meetings can be determined by the individual supervisors, but we suggest a slightly shorter interval between the first and second meeting, extending the intervals throughout the process, as the workload is likely to accumulate with time. For each session, the students are encouraged to assign a chairperson who is to assume responsibility for the structure and time planning.

\section{The 0\% Meeting: The Overview Exercise} Preparation for the $0 \%$ Meeting

The first meeting, also called the $0 \%$ meeting, should be scheduled as soon as possible after the course starts so as to maximize the time allocated to focused writing. To

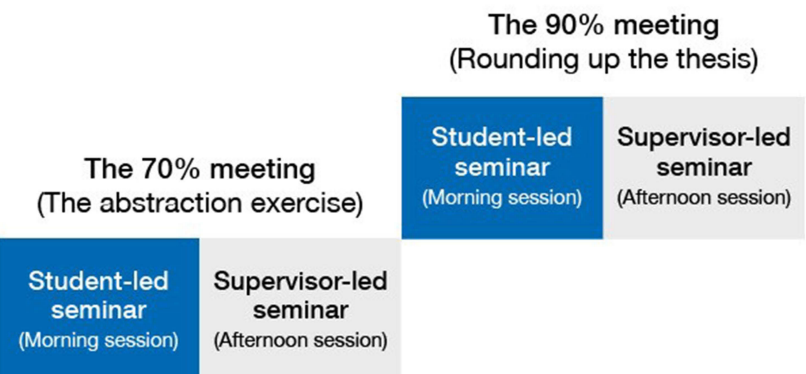

Student-led seminar seminar (Afternoon session)

Figure 2 An overview of ESRA supervision step-by-step. 
prepare for this meeting, the students should re-read their own project plans, including the examiner's comments.

\section{Morning Session}

First, supervisors introduce the ESRA model schedule. It is vital that the students be well informed about the tenets of the model as well as that all feedback be general and primarily given verbally during the meetings. This will push the students to be responsible for applying this verbal feedback in their own work. Written feedback might be given on occasion, but in a separate document and not as comments on the thesis manuscript. This is to encourage students to think critically and evaluate in what way the feedback is relevant to their own texts, as well as to keep them from only mechanically revising their texts based on the supervisors' comments. Hence, the students themselves are responsible for writing down the suggestions and recommendations from the feedback.

After the introduction of the model and schedule, the supervisors prepare the group for today's task and exercise. The first part of the morning session will be allocated to a student-led critical discussion around each pair's project plan and the examiner's comments. In the second part, the students work with the overview exercise. The overview exercise involves each pair "swapping" their thesis aim/ objective with another pair, thus each pair works with a "borrowed" aim. The exercise is to identify possible headings for the introduction, results and, if time permits, discussion sections. The purpose of this exercise is to use the strength of another pair's "fresh gaze" and to help the owner of the aim to become more broad-minded. In the afternoon session, each pair should be prepared to present an outline of headings relevant to the aim by starting with the sentence "The aim of this project has been to ..."

\section{Afternoon Session}

The afternoon starts with students presenting their results from the overview exercise. Each presentation is followed by comments from the other students, supervisors, and owners of the work (in that order). Subsequently, a general discussion of the required structure of a degree project is undertaken. After all of the presentations, areas of discussion based on the questions raised during the morning session are discussed with the attending supervisors.

\section{The 40\% Meeting: The Body Text Exercise \\ Preparation for the $40 \%$ Meeting}

In preparing for the $40 \%$ meeting, the students are to focus on their methods section in their degree project. Fortyeight hours prior to the meeting, each pair should make their methods section available to the supervisors and peers. According to the planned schedule, each pair will read another pair's methods section. When reading, the students should have the following questions in mind: Are all the headings included in the methods section according to the local thesis guidelines? Is each methods section clearly described? Are the different sections overlapping or are there any contradictions? Are the inclusion and exclusion criteria relevant? Are the search terms relevant to the research aim? The students should also test the other pairs' search strategy.

\section{Morning Session}

The meeting starts with the supervisors preparing for today's exercise. The first part of the morning session is to be allocated to a student-led critical discussion around each pair's methods section (initiated by the readers). In the second part, the students work with the body text exercise. This involves reading a given research aim and given excerpts from six articles. The students should work in pairs and analyze the excerpts, formulate a theme, and write a summary. The purpose of the exercise is to practice the handicraft of formulating a body text based on excerpts from qualitative data (articles or transcribed interviews). In the afternoon session, each pair should be prepared to present their results from the exercise.

\section{Afternoon Session}

The afternoon session starts with students presenting their results from the body text exercise. Each presentation is followed by comments from the other students, supervisors, and owners of the work (in that order). It is then appropriate to have a joint discussion about how to formulate a body text in general. Subsequently, areas of discussion based on questions raised during the morning session are discussed with the attending supervisors. The supervisors have read all methods sections and will provide general suggestions for improvements. 


\section{The 70\% Meeting: The Abstraction Exercise}

\section{Preparation for the $70 \%$ Meeting}

Preparing for the $70 \%$ meeting, the students are to focus on their results section, based on what they have learned from the body text exercise. They will also work on completing the introduction/background section. Forty-eight hours prior to the meeting, each pair is to make their results section available to the supervisors and peers. According to the planned schedule, each pair is to read another pair's results and introduction/background section. When reading, the students should focus on questions such as: Do the results address the research aim? How can the results section be improved? Are the themes/categories mutually exclusive?

\section{Morning Session}

The meeting starts with the supervisors preparing today's exercise. The first part of the morning should be allocated to a student-led critical discussion around each pair's results section (initiated by the readers). In the second part of the morning, the students will work with the abstraction exercise. This exercise involves "illustrating the results" and is carried out by either drawing on a large piece of paper, or by using a computerized drawing program or pictures/symbols from the internet (or other creative solutions), to visually illustrate their results. The purpose of this exercise is to help students gain a holistic perspective on their work as well as to discover new perspectives, concepts, headings, and patterns in their work. Further, the demand to choose what is most important to illustrate in their work should give clues about a suitable title for the project, or headings for the discussion section. The students should prepare to present their work using the sentence "My results show that ..."

\section{Afternoon Session}

The afternoon starts with the students presenting their results from the abstraction exercise. Each presentation is followed by comments from the other students, supervisors, and owners of the work (in that order). After all presentations, a joint brief discussion is held about the value of abstracting one's results. Subsequently, areas of discussion based on questions raised during the morning session are discussed with the attending supervisors. The supervisors have read the results sections and will provide general suggestions for improvements.

\section{The $90 \%$ Meeting: Rounding Up the Thesis}

Preparation for the $90 \%$ Meeting

Preparing for the $90 \%$ meeting, the students are to focus on their discussion section, based on what they have learned from the abstraction exercise. They should also work on completing the results section. Forty-eight hours prior to the meeting, each pair is to make their results section available to the supervisors and peers. According to the planned schedule, each pair is to read another pair's full thesis. When reading, the students should focus on questions such as: Is there a "main thread" in the work? Is there a relevant rationale for the study? Is there something missing in the introduction or discussion?

\section{Morning Session}

The morning session starts with supervisors preparing the students for today's task. This session does not have an exercise; instead the students are to give each other feedback on the language, formatting and referencing as well as the overall impression of the thesis.

\section{Afternoon Session}

In the afternoon session, questions and unclarified issues that came up in the morning session are to be brought up and discussed. Preparing for this session, the supervisors have read all the thesis drafts superficially and prepared general comments according to the IMRAD (Introduction, Methods, Results, And Discussion) structure, which will be provided orally. In addition, the supervisors have read the full thesis a more focused manner and prepared written comments in a separate document.

\section{Discussion}

The ESRA model departs from previously theorized supervision models in that it emphasizes dialogism, partnership between students and supervisors, and a contractual relationship where the supervisor offers direction without "taking over" responsibility for the degree project, ${ }^{10,11}$ all of which relates to a constructivist philosophy of education. ${ }^{16}$ We argue that this model not only benefits students by enabling them to achieve autonomy through development of their skills in reflective and critical thinking and problem solving, but also eases supervisors' workload. We will now discuss how and why the ESRA model may be suitable for a new generation of nursing students and supervisors. 


\section{Empowering Students to Assume Responsibility: Various Methods of Feedback}

In much "traditional" supervision, such as in the teaching model, ${ }^{10}$ the responsibility of the supervisor is to comment directly in the students' texts. When supervisors make comments on certain text passages and not others, it implies that those not commented on are "good to go," which weakens the emphasis on the students continuously assuming responsibility for improving the text. Instead the responsibility is rather located with the supervisor and his or her ability to detect poor formulations. In an analysis of teachers' written responses on students' texts, Otnes and Solheim ${ }^{17}$ found that teacher comments pointed out errors in need of correction or demanded rewrites or elaboration sometimes using very vague language with hidden intentions. We argue that this hinders students' autonomy and ability to fully "own" their work. With the ESRA model, we emphasize a shift in the responsibility for improving the text from the supervisor to the student, a shift meant to empower students. By refraining from providing any written feedback directly in the text, as suggested in the ESRA model, the students are pushed to master their own degree project and its development. This enables the responsibility to be transferred back to the rightful owner of the project, namely the students. In this sense, the ESRA model helps supporting and empowering students to develop their texts in their own style and manner.

Further, the ESRA model suggests a more diverse responsibility for the task of providing feedback, as one important component of the model is the emphasis on "peer learning," including feedback provided by fellow students. A recent study found that students reported several benefits of peer reviewing: a deeper understanding of the discipline, enhanced knowledge by virtue of being a reviewer, and increased ability to identify strengths and weaknesses in others' text as well as in their own. ${ }^{18}$ Using the ESRA model increases opportunities for students to develop these abilities, because they become familiar with reading and offering critical comments on their peer's work, which equips them with skills in critical thinking and reflection, skills that are useful in all aspects of their future, including their professional lives as nurses. Thus, the peer learning process both teaches students to receive and value feedback from equals, which helps them understand the value of the "peer-review" process in academia and develop their reflective thinking, and teaches them the ability to collaborate with fellow nurses. This can be seen as co-construction of knowledge, and is empowering.

\section{Escorting Students into Responsibility and Becoming Active Acquirers of Knowledge} The word "escorting", which refers to "going with a person and making sure he or she arrives safely" (Cambridge Dictionary, n.d.), is meant to describe the process of guiding, but not carrying, students to their intended goal. The conceptual basis for guided learning is that learners advance their learning guided by more experienced partners as well as sources such as tools, texts and other artifacts. ${ }^{19}$ In this sense, we believe that guiding or "escorting" students complements the supervising models described by Dysthe ${ }^{10}$ and Gatfield ${ }^{11}$ by emphasizing the teaching model, the partnership model and the apprenticeship model. Thus, escorting students fills the gap emphasized by Frenk et $\mathrm{al}^{12}$ by putting the educational focus on promoting the reasoning and communication skills of healthcare professionals in the 21th century. When escorting students, it is important that supervisors be prepared to relinquish their responsibility for degree project production and to see the final product as a result of the student's work, not the supervisor's. As a direct consequence of supervisors relinquishing this responsibility, students are pushed to embrace it. Silén and Johansson ${ }^{2}$ suggested that students' degree projects not only provide an opportunity to receive a professional qualification as a nurse, but also to gain a Bachelor's degree in nursing. We postulate that the embraced responsibility achieved through the production of the degree project using the ESRA model fuels students' forthcoming professional nursing work by strengthening their autonomy and sovereignty. Also Lundgren and Robertsson ${ }^{20}$ found that nurses reported utilizing the knowledge and skills they had earned during degree project writing in their nursing practice. They stated that their learned critical approach to the research process had developed their analytical and critical-thinking skills. It had also made them aware of other perspectives, including those of patients and relatives. To that end, the ESRA model extends the view of didactics according to which supervisors provide students with knowledge, focusing on students' ability to learn and adapt to our changing world.

Further, escorting students into increased responsibility and autonomy treats them as active acquirers of knowledge. In this sense, the ESRA model reinforces the notion 
that education is not limited to learning about others' research, but includes "learning to do research" through the handicraft of the research methods themselves ( $\mathrm{cf}^{21}$ ). However, the ESRA model also involves "learning in research mode",21 (p. 21), as students' degree projects result from their own developed research questions, and their work becomes highly inquiry-based. Instead of treating students as passive recipients of research results, the ESRA model pushes students to actively participate in learning to usethe model, thus forming the basis of research-based and research-integrated education. ${ }^{19,21}$

\section{Not to Be Forgotten: The Need for Contextualization and Careful Planning}

While the ESRA model is a guide for thesis group supervision that provides supervisors with a certain set of activities aimed at encouraging students and escorting them into being active in and assuming responsibility for their own learning, it is not merely "recipe" that supervisors can trust the inherent validity of. Just as with many pedagogic activities, the teacher or supervisor needs to prepare and plan ahead. The protocol needs to be thoroughly reviewed by the individual supervisor, who then must contextualize and adapt it to the university guidelines regarding study pace and level. Moreover, the supervisor needs to calculate how large the study group is and determine whether the study group will have one or two supervisors. Our experience is that the supervisor must structure the supervision framework in advance, so that all students will receive an equal proportion of the formal time. For example, during the $0 \%$ meeting, the presentation of the task in the afternoon, each student pair may be given a maximum of 18 minutes, including discussion. This mitigates the risk that the last group will end up with a very narrow time window. The planning can be seen as an underlying prerequisite for the overarching structure of the ESRA model.

The ERSA model has been successfully applied in the education of students on the levels of Master and Bachelor of Science at three universities in Sweden, but only within the discipline of nursing. However, we are confident that the model can also be applied in other disciplines where students need to develop their academic writing skills, which broadens the generalizability of the model's usefulness. Another limitation is that the model has not yet been scientifically evaluated by students or supervisors, thus we know little beyond our own experience of how supervisors or students perceive the proposed protocol. Further, we know little about how the model might benefit students moving on to more advanced thesis courses or about its usefulness in regards to improving applied practical and professional skills.

Thus, we suggest that future studies should investigate the perceived applicability and utility of the ESRA model among nurses as well as students within other disciplines.

\section{Conclusion}

The ESRA model presented here is an educational protocol that is suitable for guiding students and their supervisors in a constructive interaction to achieve the learning outcomes of the degree project. The model promotes a high level of engagement and assumption of responsibility among students, while also offering a feasible structure to ensure the steps to empowerment and autonomy. More importantly, the model promotes the improvement of students' skills within a certain structure that can be repeated over and over, even after the students move beyond the student role. The proposed model constitutes one step toward fostering a generation of nurses and other professionals who have the skills and ability to adapt in the changing world of the 21 th century.

\section{Ethics}

As the present paper is solely theoretical and does not involve human subjects, the study does not require ethical approval.

\section{Acknowledgments}

We would like to acknowledge Dr. Joacim Larsen for his significant contribution to the development and establishment of the model in the higher education and Dr. Sara Stridh for assisting in implementing the model.

\section{Disclosure}

The authors report no conflicts of interest in this work.

\section{References}

1. Gallart A, Bardallo MD, de Juan M, et al. Impact of the bachelor's thesis on the nursing profession. Nurse Educ Today. 2015;35(1): 16-17. doi:10.1016/j.nedt.2014.09.011

2. Silén M, Johansson L. Aims and theoretical frameworks in nursing students' bachelor's theses in Sweden: a descriptive study. Nurse Educ Today. 2016;37:91-96. doi:10.1016/j.nedt.2015.11.020

3. Greenwood J. Critique of the graduate nurse: an international perspective. Nurse Educ Today. 2000;20(1):17-23. doi:10.1054/nedt. 2000.0424 
4. Barnacle R, Dall'Alba G. Beyond skills: embodying writerly practices through the doctorate. Stud High Educ. 2014;39(7):1139-1149. doi:10.1080/03075079.2013.777405

5. Borglin G, Fagerström C. Nursing students' understanding of critical thinking and appraisal and academic writing: a descriptive, qualitative study. Nurse Educ Pract. 2012;12(6):356-360. doi:10.1016/j. nepr.2012.04.009

6. Whitehead D. The academic writing experiences of a group of student nurses: a phenomenological study. J Adv Nurs. 2002;38 (5):498-506. doi:10.1046/j.1365-2648.2002.02211.x

7. Rega ML, Telaretti F, Alvaro R, Kangasniemi M. Philosophical and theoretical content of the nursing discipline in academic education: a critical interpretive synthesis. Nurse Educ Today. 2017;57:74-81. doi:10.1016/j.nedt.2017.07.001

8. Studer P. Reflexivity and academic writing: how supervisors deal with self-discovery in student teachers' bachelor's theses. Int J Appl Linguist. 2017;27(3):651-664. doi:10.1111/ijal.12167

9. Aguayo-González M, Leyva-Moral JM, San Rafael S, Fernandez MI, Gómez-Ibáñez R. Graduated nurses' experiences with baccalaureate thesis writing: a qualitative study. Nurs Health Sci. 2020;22(3): 563-569. doi:10.1111/nhs.12693

10. Dysthe O. Professors as mediators of academic text cultures: an interview study with advisors and master's degree students in three disciplines in a Norwegian University. Writ Commun. 2002;19 (4):493-544. doi:10.1177/074108802238010

11. Gatfield T. An investigation into $\mathrm{PhD}$ supervisory management styles: development of a dynamic conceptual model and its managerial implications. J High Educ Policy Manag. 2005;27(3):311-325. doi:10.1080/13600800500283585
12. Frenk J, Chen L, Bhutta ZA, et al. Health professionals for a new century: transforming education to strengthen health systems in an interdependent world. Lancet. 2010;376(9756):1923-1958. doi:10.10 16/s0140-6736(10)61854-5

13. Johansson L, Silén M. Research methods in nursing students' bachelor's theses in Sweden: a descriptive study. Nurse Educ Today. 2018;66:187-193. doi:10.1016/j.nedt.2018.04.006

14. Teo P. 'Stretch your answers': opening the dialogic space in teaching and learning. Learn Cult Soc Interact. 2013;2(2):91-101. doi:10. 1016/j.lcsi.2013.02.002

15. Gilstrap DL. Dialogic and the emergence of criticality in complex group processes. JCACS. 2008;6:91-112.

16. Juvova L, Neumeister P, Plischke J, Kvintova J. Reflection of constructivist theories in current educational practice. Univers $J$ Educ Res. 2015;3(5):345-349. doi:10.13189/ujer.2015.030506

17. Otnes H, Solheim R. Acts of responding. Teachers' written comments and students' text revisions. Assess Educ Princ Pol Pract. 2019;26 (6):700-720. doi:10.1080/0969594X.2019.1595524

18. Reddy K, Harland T, Wass R, Wald N. Student peer review as a process of knowledge creation through dialogue. High Educ Res Dev. 2020;1-13. doi:10.1080/07294360.2020.1781797

19. Billett S. Guided learning. In: Seel NM, editor. Encyclopedia of the Sciences of Learning. US: Springer; 2012:1403-1406.

20. Lundgren SM, Robertsson B. Writing a bachelor thesis generates transferable knowledge and skills useable in nursing practice. Nurse Educ Today. 2013;33(11):1406-1410. doi:10.1016/j.nedt.2012.10.019

21. Jenkins A, Healey M. Institutional Strategies to Link Teaching and Research. The Higher Education Academy; 2005.
Advances in Medical Education and Practice

\section{Publish your work in this journal}

Advances in Medical Education and Practice is an international, peerreviewed, open access journal that aims to present and publish research on Medical Education covering medical, dental, nursing and allied health care professional education. The journal covers undergraduate education, postgraduate training and continuing medical education

\section{Dovepress}

including emerging trends and innovative models linking education, research, and health care services. The manuscript management system is completely online and includes a very quick and fair peer-review system. Visit http://www.dovepress.com/testimonials.php to read real quotes from published authors. 